\title{
Turing Instability and Amplitude Equation of Reaction-Diffusion System with Multivariable
}

\author{
Qianqian Zheng ${ }^{1}$ and Jianwei Shen (D) $^{2}$ \\ ${ }^{1}$ School of Mathematics and Statistics, Xuchang University, Xuchang, Henan 461000, China \\ ${ }^{2}$ School of Mathematics and Statistics, North China University of Water Resources and Electric Power, Zhengzhou 450046, China \\ Correspondence should be addressed to Jianwei Shen; xcjwshen@gmail.com
}

Received 3 February 2020; Accepted 29 February 2020; Published 29 March 2020

Guest Editor: Rongwei Guo

Copyright (c) 2020 Qianqian Zheng and Jianwei Shen. This is an open access article distributed under the Creative Commons Attribution License, which permits unrestricted use, distribution, and reproduction in any medium, provided the original work is properly cited.

\begin{abstract}
In this paper, we investigate pattern dynamics with multivariable by using the method of matrix analysis and obtain a condition under which the system loses stability and Turing bifurcation occurs. In addition, we also derive the amplitude equation with multivariable. This is an effective tool to investigate multivariate pattern dynamics. The example and simulation used in this paper validate our theoretical results. The method presented is a novel approach to the investigation of specific real systems based on the model developed in this paper.
\end{abstract}

\section{Introduction}

The pattern formation was first investigated and interpreted by Alan Turing 60 years ago [1]. In general, Turing model contains two reactants: activator and inhibitor, which engage in diffusion. Recently, the study of Turing bifurcation, amplitude equation, and secondary bifurcation have paid more attention on the pattern formation [2-4], and Lee and Cho found that dynamical parameters and external periodic forcing play an important role in the shape and type of pattern formation [5]. And the robustness problem is also investigated [6]. The effects of cross-diffusion, the phenomenon in which a gradient in the concentration of one species induces other species, on pattern formation in reaction-diffusion systems have been discussed in many theoretical papers [7-14]. Regarding noise, noise is a ubiquitous phenomenon in nature and always deemed to play a very important role in the natural synthetic system [15]. Viney and Reece [16] treated noise as adaptive and suggested that applying evolutionary rigour to the study of noise is necessary to fully understand organismal phenotypes, and Shen considered the Lévy noise in the gene network $[17,18]$.
Recently, the pattern formation with three or four variables has been investigated, and it obtains promising results $[19,20]$, and $\mathrm{Xu}$ et al. made a concrete analysis with three variables [21]. As we all know that amplitude equation is a promising tool to investigate the pattern dynamics of the reaction-diffusion system $[2,22]$, however, the amplitude equation is a complex process [3], and the researcher often chose the amplitude equation [23-26] to investigate the reaction-diffusion system. In conclusion, spatial patterns in reaction-diffusion systems have attracted the interest of experimentalists and theorists during the last few decades. But, these previous works did not give a general method to define Turing instability and derive the amplitude equation with $n$ variables.

Besides, the study of patterns can offer useful information on the underlying processes causing possible changes in the system. In order to better understand the reaction-diffusion model, first, we propose to study the Turing instability with $n$ variables by matrix theory [27]. In addition, we also derive the amplitude equation by using the standard multiple scale analysis $[28,29]$ which provides a way to investigate the mechanism of pattern formation.

The paper is organized as follows. In Section 2, we give the general reaction diffusion with multivariable and derive 
the condition of Turing instability. In Section 3, we derive the amplitude equation from the reaction-diffusion system with multivariable. In Section 4, we utilize an example to illustrate the application of these ideas. The simulation validates our theoretical results. Finally, we summarize our results and conclusion.

\section{Turing Instability with Multivariate}

For a multivariate reaction-diffusion system, we have

$$
\frac{\partial \mathbf{u}}{\partial t}=\mathbf{f}(\mathbf{u})+\mathbf{D} \nabla^{2} \mathbf{u}, \quad \mathbf{u}=\left(u_{11}, \ldots, u_{n n}\right),
$$

where the function $\mathbf{f}(\mathbf{u})$ specifies dynamics of the interaction of the species and $\mathbf{D}$ is the diffusion parameter diagonal matrix.

Also, we can obtain the following linear system at equilibrium $\mathbf{u}=\mathbf{0}$ from (1):

$$
\frac{\partial \mathbf{u}}{\partial t}=\mathbf{A} \mathbf{u}+\mathbf{D} \nabla^{2} \mathbf{u}
$$

where $A$ is the Jacobian matrix.

As we all know that the stability of a system depends on the sign of the real part of eigenvalue [23], for coefficient matrix $\mathbf{A}$, there is a nonsingular matrix $\mathbf{P}$ subject to $\mathbf{A}=\mathbf{P}^{-1} \mathbf{J P}$, and $\mathbf{J}$ is a Jordan form [27]. Also, we have

$$
\begin{aligned}
|\lambda \mathbf{I}-\mathbf{A}|=|\lambda \mathbf{I}-\mathbf{J}| & =\left|\begin{array}{cccc}
\lambda-\mathbf{J}_{1} & 0 & 0 & 0 \\
0 & \lambda-\mathbf{J}_{2} & 0 & \vdots \\
0 & 0 & \lambda-\mathbf{J}_{3} & 0 \\
0 & \cdots & \cdots & \lambda-\mathbf{J}_{n}
\end{array}\right| \\
& =\left(\lambda+\lambda_{1}\right)\left(\lambda+\lambda_{2}\right) \cdots\left(\lambda+\lambda_{n}\right)=0,
\end{aligned}
$$

where

$$
\mathbf{J}=\left(\begin{array}{ccc}
\mathbf{J}_{1} & 0 & \vdots \\
0 & \mathbf{J}_{2} & \vdots \\
\cdots & \cdots & \mathbf{J}_{i}
\end{array}\right),
$$

where

$$
\mathbf{J}_{k}=\left(\begin{array}{cccc}
\lambda_{k} & 1 & 0 & 0 \\
0 & \lambda_{k} & 1 & \vdots \\
0 & 0 & \lambda_{k} & 1 \\
0 & \cdots & \cdots & \lambda_{k}
\end{array}\right) \text {, }
$$

where $-\lambda_{i}$ is the eigenvalue and has a negative real part which means stable without diffusion. In addition, we can get the condition of stability by Routh-Hurwitz criteria.

In the standard way, we assume that $\mathbf{u}$ takes the form

$$
\mathbf{u}=\mathbf{c} e^{\lambda_{k} t+i k r},
$$

and obtains the characteristic equation from system (1.2) as follows:

$$
\left|\lambda \mathbf{I}-\mathbf{A}+\mathbf{D} k^{2}\right|=\left|\lambda \mathbf{I}-\mathbf{J}-\mathbf{D} k^{2}\right|=\lambda^{n}+a_{1} \lambda^{n-1}+\cdots+a_{n-1} \lambda+a_{n}=0,
$$

and there is at least $\lambda>0$ which exists when $a_{n}<0$ based on Routh-Hurwitz criteria.

Now, we obtain the definition of Turing instability with $n$ variables.

Theorem 1. Turing instability occurs when $a_{n}<0$.

In addition, we obtain the critical condition of Turing instability. Assume $x=k^{2}$ and $p(x)=x^{n}+p_{1} x^{n-1}$ $+\cdots+p_{n}, p^{\prime}(x)=0$ has $n-1$ roots, and $x_{i}, i=1, \ldots, n-1$ and can get the minimum value $p\left(x_{c}>0\right)$ [30]. We can obtain the critical condition of Turing instability from $a_{n}\left(k_{c}^{2}\right)=0$.

\section{Amplitude Equation with Multivariable}

In this paper, we continue to study the system with $n$ variables. In the following, we use multiple scale analysis to derive the amplitude equations.

The solutions of systems can be expanded as

$$
c=c_{0}+Z_{i} e^{i k_{i} r}+\text { c.c. }, \quad i=1,2,3 .
$$

And system (1) can be written as

$$
\frac{\partial \mathbf{c}}{\partial t}=\mathbf{L c}+N(\mathbf{c}, \mathbf{c})
$$

where $\mathbf{c}=\mathbf{u}$ is the variable, $\mathbf{L}=\mathbf{A}+\mathbf{D} \nabla^{2}$ is the linear operator, $N=N_{1} \mathbf{u}^{2}+N_{2} \mathbf{u}^{3}$ is the nonlinear term, $N_{1} \mathbf{u}^{2}$ is all the twice term, and $N_{2} \mathbf{u}^{3}$ is all the triple term.

We need to investigate the dynamical behavior when $\gamma$ is close to $\gamma_{c}$, and then we expand $\gamma$ as

$$
\gamma_{c}-\gamma=\varepsilon \gamma_{1}+\varepsilon^{2} \gamma_{2}+\cdots
$$

where $\gamma_{c}$ is the critical value and $\varepsilon$ is a small enough parameter.

We expand $\mathbf{c}$ and $N$ as the series form of $\varepsilon$ :

$$
\begin{aligned}
\mathbf{c} & =\mathbf{u}_{1} \varepsilon+\mathbf{u}_{2} \varepsilon^{2}+\cdots, \\
N & =N_{1} \mathbf{u}_{1}^{2} \varepsilon^{2}+\left(N_{1} \mathbf{u}_{1} \mathbf{u}_{2}+N_{2} \mathbf{u}_{1}^{3}\right) \varepsilon^{3}+o\left(\varepsilon^{4}\right) .
\end{aligned}
$$

Linear operator $L$ can be expanded as

$$
\mathbf{L}=\mathbf{L}_{c}+\left(\gamma_{c}-\gamma\right) M .
$$

Let

$$
\begin{aligned}
& T_{0}=t, \\
& T_{1}=\varepsilon t, \\
& T_{2}=\varepsilon^{2} t, \ldots .
\end{aligned}
$$

$T_{i}$ is a dependent variable, and amplitude is a slow variable. For the derivation of time, we have that

$$
\frac{\partial W}{\partial t}=\varepsilon \frac{\partial W}{\partial T_{1}}+\varepsilon^{2} \frac{\partial W}{\partial T_{2}}+\cdots
$$

Substituting the above equations into (1) and expanding (1) according to different orders of $\varepsilon$, we can obtain three equations as follows: 


$$
\begin{aligned}
& \varepsilon: \mathbf{L}_{c} \mathbf{u}_{1}=0 \\
& \varepsilon^{2}: \mathbf{L}_{c} \mathbf{u}_{2}=\frac{\partial}{\partial T_{1}} \mathbf{u}_{1}-\gamma_{1} M \mathbf{u}_{1}-N_{1} \mathbf{u}_{1}^{2} \\
& \varepsilon^{3}: \mathbf{L}_{c} \mathbf{u}_{3}=\frac{\partial}{\partial T_{1}} \mathbf{u}_{2}+\frac{\partial}{\partial T_{2}} \mathbf{u}_{1}-\gamma_{1} M \mathbf{u}_{2}-\gamma_{2} M \mathbf{u}_{1}-N_{1} \mathbf{u}_{1} \mathbf{u}_{2}-N_{2} \mathbf{u}_{1}^{3}
\end{aligned}
$$

We first consider the case of the first order of $\varepsilon$. Since $L_{c}$ is the linear operator of the system close to the onset, $\mathbf{u}_{1}$ is the linear combination of the eigenvectors that corresponds to the eigenvalue zero. Since that

$$
\mathbf{u}_{1}=\mathbf{b} W_{i} e^{i k_{i} r}+c . c
$$

where $\mathbf{L}_{c} \mathbf{b}=0$, nonzero root exists [27]

Now, we consider the case of $\varepsilon^{2}$ and the zero eigenvectors of operator $\mathbf{L}_{c}^{+}, \exists \mathbf{b}^{+}$s.t.:

$$
\mathbf{L}_{c}^{+} \mathbf{b}^{+}=0 \text {. }
$$

By investigating $e^{i k_{1} r}$ only in the following, another case we can get is by changing subscript which is not described in detail here. It can be obtained from the orthogonality condition that

$$
\mathbf{b}^{+T} \mathbf{b} \frac{\partial}{\partial T_{1}} W_{1}=\mathbf{b}^{+T} M \mathbf{b} W_{1}+\left(\mathbf{b}^{+T} \mathbf{b}^{2}\right) N_{1} \overline{W_{2} W_{3}} .
$$

By using the same methods, one has

$$
\begin{aligned}
\mathbf{u}_{2}= & a_{0}+a_{i} Z_{i} e^{i k_{i} r}+a_{i i} Z_{i i} e^{i 2 k_{i} r}+a_{12} Z_{12} e^{i\left(k_{1}-k_{2}\right) r} \\
& +a_{23} Z_{23} e^{i\left(k_{2}-k_{3}\right) r}+a_{31} Z_{31} e^{i\left(k_{3}-k_{1}\right) r}+\text { c.c. }
\end{aligned}
$$

For the case of $\varepsilon^{3}$, we have that

$$
\mathbf{L}_{c} \mathbf{u}_{2}=0 \text {. }
$$

We can obtain all the coefficients and $a_{i}=\mathbf{b}$.

Using the Fredholm solubility condition again, we can obtain

$$
\begin{aligned}
\left(\mathbf{b}^{+T} \mathbf{b}\right) \frac{\partial Z}{\partial T_{1}}+\left(\mathbf{b}^{+T} \mathbf{b}\right) \frac{\partial Z}{\partial T_{2}}= & \gamma_{1} \mathbf{b}^{+T} M \mathbf{b} Z_{1}+\gamma_{2} \mathbf{b}^{+T} M \mathbf{b} W_{1} \\
& +\mathbf{b}^{+T} \mathbf{b}^{2} N_{1}\left(\overline{Z_{2} W_{3}}+\overline{Z_{3} W_{2}}\right) \\
& +\mathbf{b}^{+T} \mathbf{b}^{3} N_{2}\left(|W|_{1}^{2}+\left|W_{2}\right|^{2}+\left|W_{3}\right|^{2}\right) W_{1}
\end{aligned}
$$

and then we substitute systems (18) and (21) into (14) for simplification, in which we can obtain

$$
\begin{aligned}
\left(\mathbf{b}^{+T} \mathbf{b}\right) \frac{\partial W_{1}}{\partial t}= & \left(\gamma_{c}-\gamma\right) W_{1}+\mathbf{b}^{+T} \mathbf{b}^{2} N_{1} \overline{W_{2} W_{3}} \\
& +\mathbf{b}^{+T} \mathbf{b}^{3} N_{2}\left(|W|_{1}^{2}+\left|W_{2}\right|^{2}+\left|W_{3}\right|^{2}\right) W_{1} .
\end{aligned}
$$

\section{Method}

In the following, we consider the Turing instability of a system with 3 variables:

$$
\begin{aligned}
& \frac{\partial u}{\partial t}=\frac{k_{0} u}{1+k_{0} u}-\mu u+d_{1} \nabla^{2} u, \\
& \frac{\partial v}{\partial t}=\frac{k_{1} u+k_{2} v}{1+k_{1} u+k_{2} v}-\mu v+d_{2} \nabla^{2} v, \\
& \frac{\partial w}{\partial t}=\frac{k_{3} u+k_{4} v+k_{5} w}{1+k_{3} u+k_{4} v+k_{5} w}-\mu w+d_{3} \nabla^{2} w,
\end{aligned}
$$

and we obtain the Jacobian matrix at equilibrium $\left(u_{0}, v_{0}, w_{0}\right)=(0,0,0)$ :

$$
A=\left(\begin{array}{ccc}
k_{0}-\mu & 0 & 0 \\
k_{1} & k_{2}-\mu & 0 \\
k_{3} & k_{4} & k_{5}-\mu
\end{array}\right) .
$$

The characteristic equation is

$$
|\lambda I-A|=\left(\lambda-k_{0}+\mu\right)\left(\lambda-k_{2}+\mu\right)\left(\lambda-k_{5}+\mu\right)=0 .
$$

The system is stable without diffusion when $\mu-k_{0}<0, \mu-k_{2}<0$ and $\mu-k_{5}<0$, that is to say it is stable when $\mu<\min \left(k_{0}, k_{2}, k_{5}\right)$.

Then, the Jacobian matrix with diffusion is given as follows:

$$
B=\left(\begin{array}{ccc}
k_{0}-\mu-d_{1} k^{2} & 0 & 0 \\
k_{1} & k_{2}-\mu-d_{2} k^{2} & 0 \\
k_{3} & k_{4} & k_{5}-\mu-d_{3} k^{2}
\end{array}\right) .
$$

The characteristic equation is

$$
|\lambda I-B|=\lambda^{3}+a \lambda^{2}+b \lambda+c=0,
$$

where

$$
\begin{aligned}
a= & \left(d_{3}+d_{1}+d_{2}\right) k^{2}-k_{0}-k_{5}+3 \mu-k_{2}, \\
b= & \left(d_{2} d_{3}+d_{1} d_{3}+d_{1} d_{2}\right) k^{4}+\left(-d_{1} k_{2}-k_{0} d_{3}+2 \mu d_{3}+2 \mu d_{2}\right. \\
& \left.-k_{0} d_{2}-k_{2} d_{3}-d_{2} k_{5}-d_{1} k_{5}+2 d_{1} \mu\right) k^{2} \\
& -2 \mu k_{2}+3 \mu^{2}-2 \mu k_{5}-2 k_{0} \mu+k_{0} k_{2}+k_{2} k_{5}+k_{0} k_{5}, \\
c= & a_{5} k^{6}+b_{5} k^{4}+c_{5} k^{2}+d_{5},
\end{aligned}
$$

where

$$
\begin{aligned}
a_{5}= & d_{1} d_{2} d_{3}, \\
b_{5}= & \mu d_{2} d_{3}-k_{0} d_{2} d_{3}-d_{1} k_{2} d_{3}+d_{1} \mu d_{3}-d_{1} d_{2} k_{5}+d_{1} d_{2} \mu, \\
c_{5}= & -\mu d_{2} k_{5}-d_{1} \mu k_{5}+\mu^{2} d_{2}-k_{0} d_{2} \mu-d_{1} k_{2} \mu-\mu k_{2} d_{3} \\
& +k_{0} d_{2} k_{5}+d_{1} \mu^{2}+d_{1} k_{2} k_{5}+\mu^{2} d_{3}+k_{0} k_{2} d_{3}-k_{0} \mu d_{3}, \\
d_{5}= & -\mu^{2} k_{5}+k_{0} \mu k_{5}+\mu k_{2} k_{5}+\mu^{3}+k_{0} k_{2} \mu-k_{0} k_{2} k_{5}-\mu^{2} k_{2}-k_{0} \mu^{2} .
\end{aligned}
$$

For convenience, we assume $x=k^{2}$, and then $f(x)=$ $a_{5} x^{3}+b_{5} x^{2}+c_{5} x+d_{5} \quad$ and $\quad f^{\prime}(x)=3 a_{5} x^{2}+2 b_{5} x+c_{5}$ which have two roots $x_{12}=-b_{5} \pm \sqrt{b_{5}^{2}-3 a_{5} c_{5}}$. We know $f(x) \geq f\left(x_{1}\right), k_{c}^{2}=x_{1}=-b_{5}+\sqrt{b_{5}^{2}-3 a_{5} c_{5}}$, and the critical value is $f\left(x_{1}\right)=0$. 


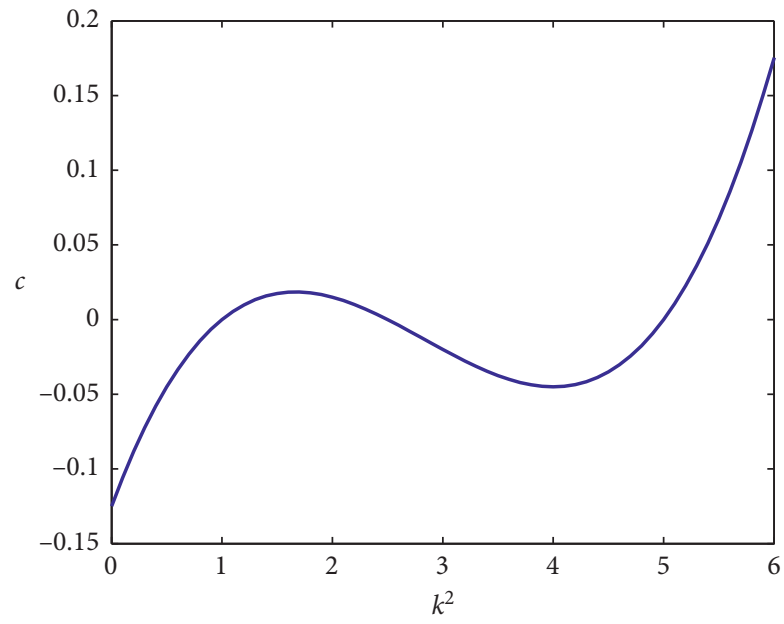

(a)

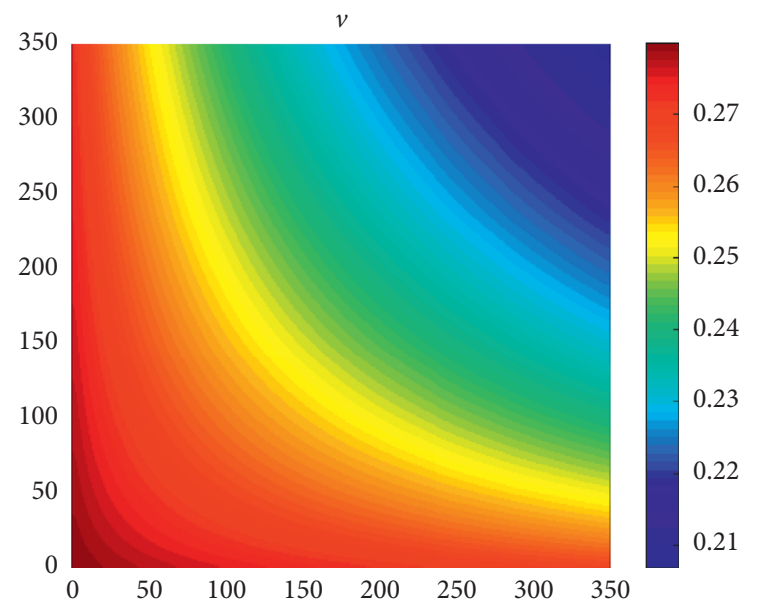

(c)

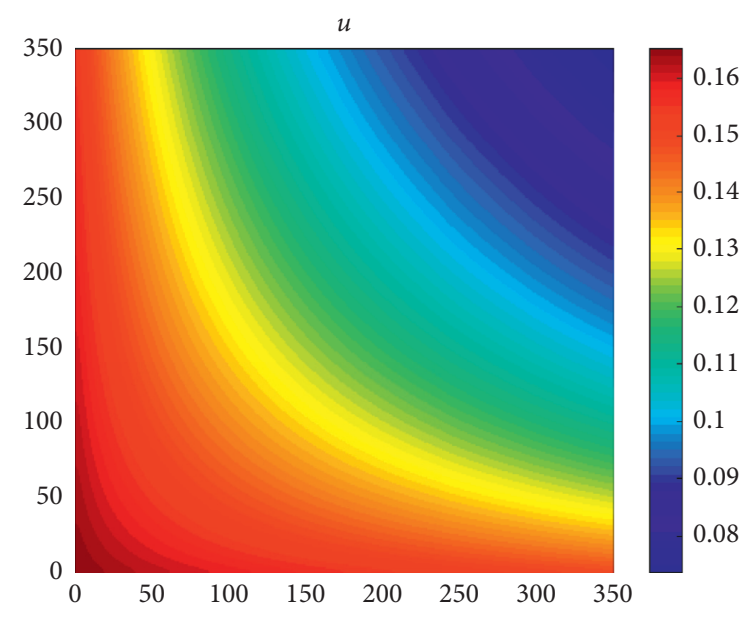

(b)

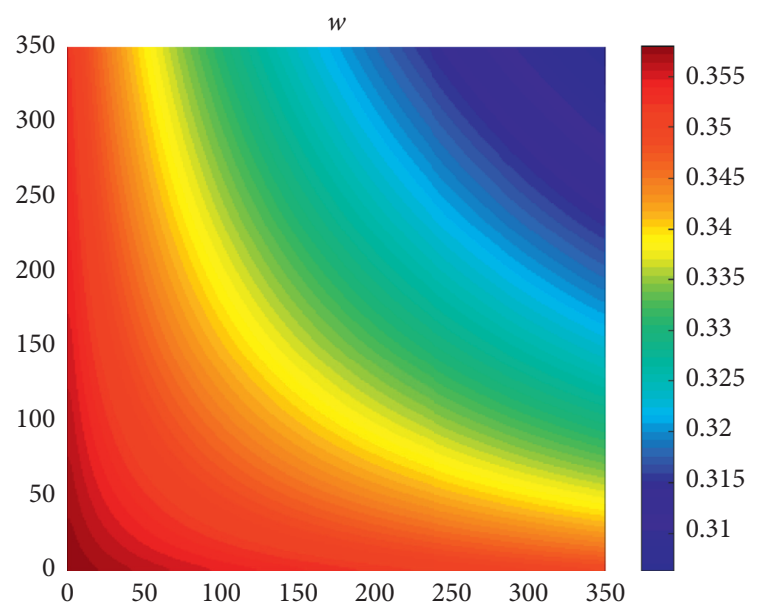

(d)

FIgURe 1: Dispersion curve and rainbow pattern.

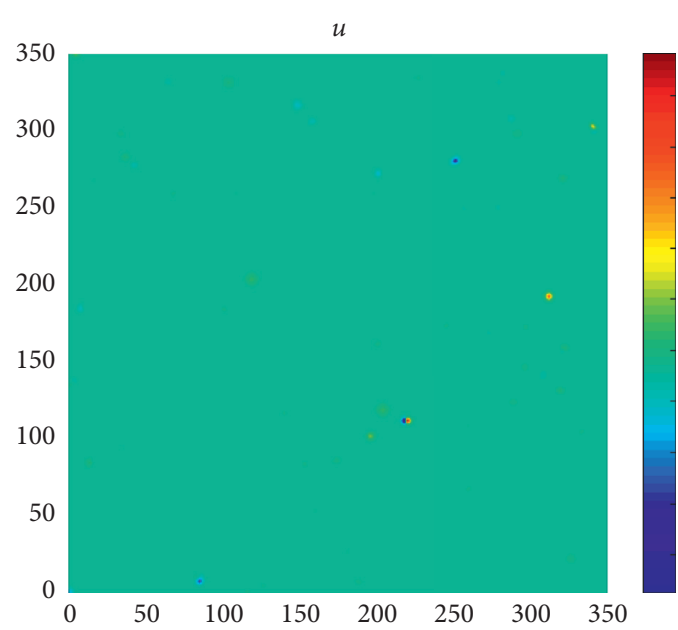

(a)

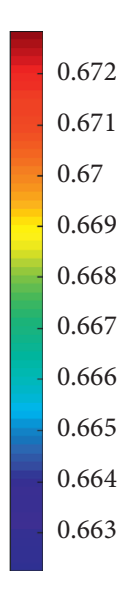
0.672
0.671
0.67
0.669
0.668
0.667
0.666
0.665
0.664
0.663

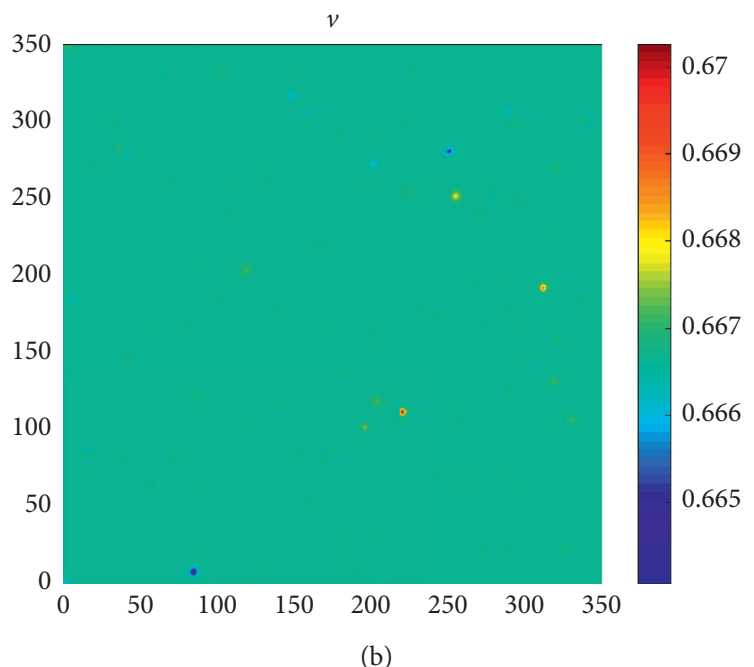

(b)

FIgURE 2: Continued. 


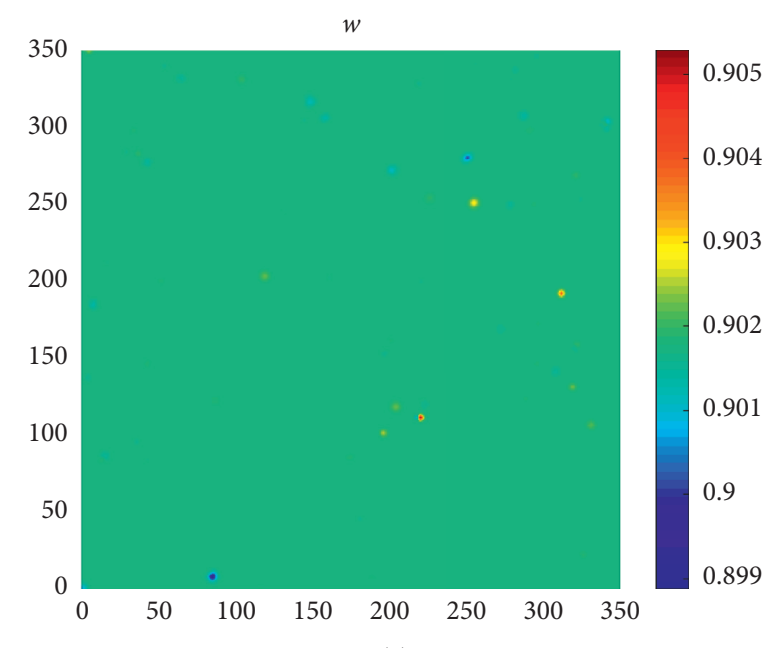

(c)

FIgURE 2: Spot pattern occurs.

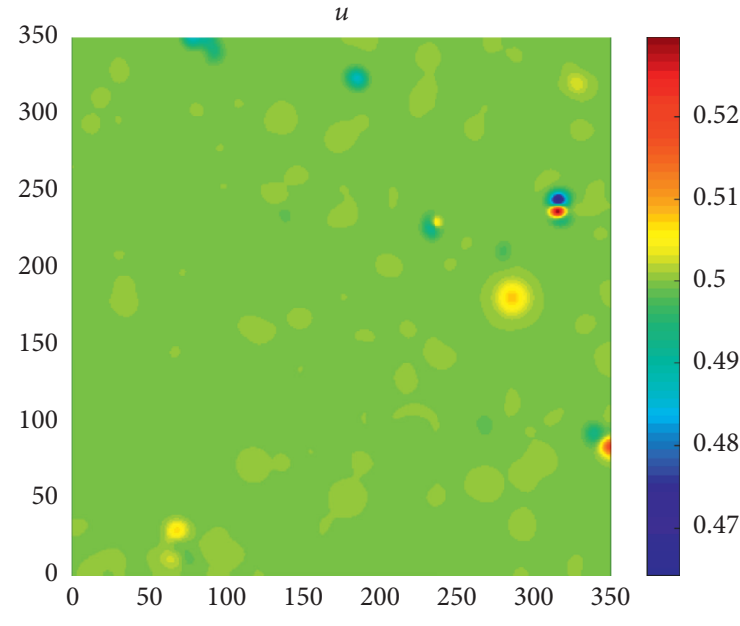

(a)

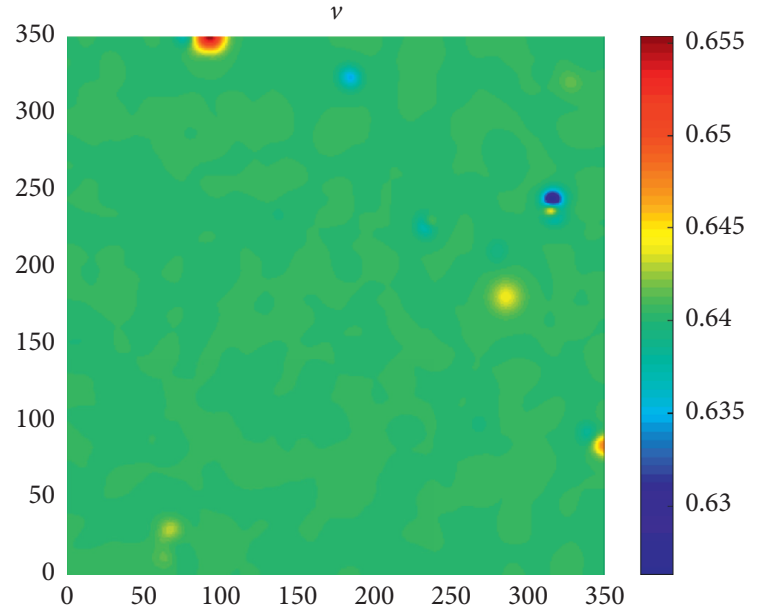

(b)

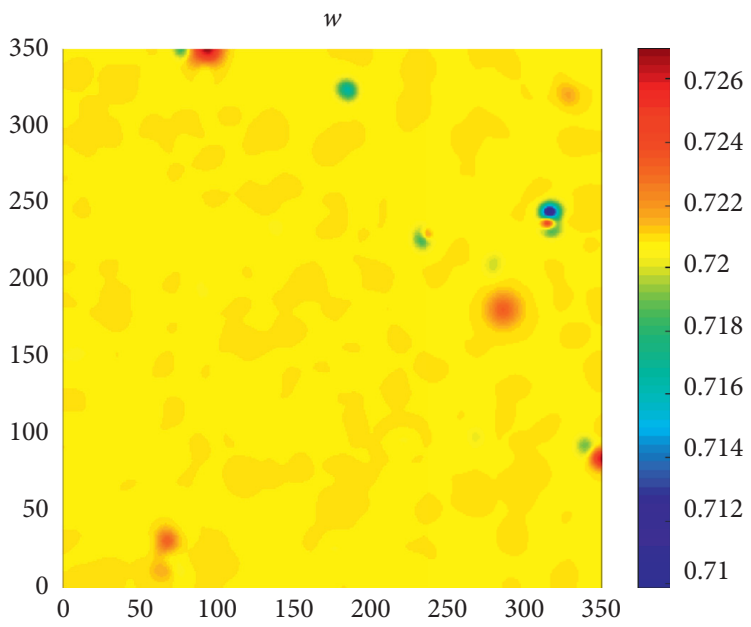

(c)

Figure 3: Nebulous pattern occurs. 
We get $k_{c}^{2}=0.12, c=-0.1022$ when $k_{0}=2, k_{1}=1, k_{2}=$ $2, k_{3}=1, k_{4}=1, k_{5}=2, d_{1}=0.1, d_{2}=0.2, d_{3}=0.5$, and $\mu=1.5$ with the perturbation $1 /((X+10)(Y+20))$ which means Turing instability, and then the rainbow stripe pattern (Figures 1(b)-1(d)) and dispersion curve occur (Figure 1(a)). In addition, spot pattern occurs (Figure 2) when $k_{0}=3, k_{1}=1, k_{2}=2, k_{3}=3, k_{4}=4, k_{5}=5, d_{1}=0.1$, $d_{2}=0.2, d_{3}=0.3, \mu=1$ with the perturbation $\sin \left(X^{2}+Y^{2}\right)$, and nebulous pattern occurs (Figure 3 ) when $k_{0}=2, k_{1}=1$, $k_{2}=2, k_{3}=1, k_{4}=1, k_{5}=2, d_{1}=1, d_{2}=2, d_{3}=1, \mu=1$ with the perturbation $\sin \left(X^{2}+Y^{2}\right)$.

\section{Conclusion}

In this article, we present the theoretical analysis and numerical simulation of the Turing instability with multivariable. It is found that the reaction-diffusion systems with multivariable have rich spatial dynamics by performing a series of numerical simulations. We also give a general method to derive the amplitude equation with multivariable in theory which can be used to solve some problems about pattern formation with multiple variables in the further study. In addition, the mechanism of pattern formation with multiple variables is on the way and can be derived based on the above theory in this paper; however, it is a very complex process, and we will investigate it in the future.

\section{Data Availability}

No data were used to support this study.

\section{Conflicts of Interest}

The authors declare that there are no conflicts of interest.

\section{Acknowledgments}

This work was supported by the National Natural Science Foundation of China (11772291), Young Talent Support Project of Henan Province (2020HYTP012), and Key Program of Xuchang University (2020ZD012).

\section{References}

[1] A. Turing, "The chemical basis of morphogenesis," Philosophical Transactions of the Royal Society B, vol. 237, pp. 37-72, 1952.

[2] H. Liu and W. Wang, "The amplitude equations of an epidemic model," Science Technology and Engineering, vol. 10, no. 8, pp. 1929-1933, 2010.

[3] A. K. Dutt, "Amplitude equation for a diffusion-reaction system: the reversible Sel'kov model," AIP Advances, vol. 2, no. 4, Article ID 042125, 2012.

[4] Q. Zheng and J. Shen, "Dynamics and pattern formation in a cancer network with diffusion," Communications in Nonlinear Science and Numerical Simulation, vol. 27, no. 1-3, pp. 93-109, 2015.

[5] I. Lee and U. I. Cho, "Pattern formations with turing and hopf oscillating pattern," Bulletin of the Korean Chemical Society, vol. 21, no. 12, pp. 1213-1216, 2000.
[6] P. K. Maini, T. E. Woolley, R. E. Baker et al., “Turing's model for biological pattern formation and the robustness problem," Interface Focus, vol. 2, no. 4, pp. 487-496, 2012.

[7] V. K. Gaffney, "Epstein IR, cross-diffusion and pattern formation in reaction-diffusion systems," Physical Chemistry Chemical Physics, vol. 11, pp. 897-912, 2008.

[8] D. Fanelli, C. Cianci, and F. D. Patti, "Turing instabilities in reaction-diffusion systems with cross diffusion," The European Physical Journal B, vol. 86, no. 4, pp. 1-8, 2013.

[9] J. Shi, Z. Xie, and K. Little, "Cross-diffusion induced instability and stability in reaction-diffusion systems," Journal of Applied Analysis and Computation, vol. 1, no. 1, pp. 95-119, 2011.

[10] Z. Liu, J. Shen, S. Cai, and F. Yan, MicroRNA Regulatory Network: Structure and Function, Springer, Berlin, Germany, 2018.

[11] Q. Zheng, J. Shen, and Z. Wang, "Pattern formation and oscillations in reaction-diffusion model with p53-Mdm2 feedback loop," International Journal of Bifurcation and Chaos, vol. 29, no. 14, Article ID 1930040, 2019.

[12] Q. Zheng, J. Shen, and Z. Wang, "Pattern dynamics of the reaction-diffusion immune system," PLoS One, vol. 13, no. 1, Article ID e0190176, 2018.

[13] Q. Zheng, Z. Wang, and J. Shen, "Pattern dynamics of network-organized system with cross-diffusion," Chinese Physics B, vol. 26, no. 2, Article ID 020501, 2017.

[14] Q. Zheng, Z. Wang, J. Shen, and H. M. Iqbal, "Turing bifurcation and pattern formation of stochastic reaction-diffusion system," Advances in Mathematical Physics, vol. 2017, Article ID 9648538, 9 pages, 2017.

[15] M. Thattai and A. Van Oudenaarden, "Intrinsic noise in gene regulatory networks," Proceedings of the National Academy of Sciences, vol. 98, no. 15, pp. 8614-8619, 2001.

[16] M. Viney and S. E. Reece, "Adaptive noise," Proceedings of the Royal Society B: Biological Sciences, vol. 280, no. 1767, Article ID 20131104, 2013.

[17] Y. Ding, J. Shen, J. Lu, and J. Kurths, "Stochastic resonance in genetic regulatory networks under Lévy noise," EPL (Europhysics Letters), vol. 127, no. 5, p. 50003, 2019.

[18] Q. H. Zhu, J. W. Shen, and J. C. Ji, "Internal signal stochastic resonance of a two-component gene regulatory network under Lévy noise," Nonlinear Dynamics, pp. 1-4, 2020.

[19] K. Uriu and Y. Iwasa, "Turing pattern formation with two kinds of cells and a diffusive chemical," Bulletin of Mathematical Biology, vol. 69, no. 8, pp. 2515-2536, 2007.

[20] Z. Li and W. Chen, "Two dimension spatial pattern formation in a coupled autocatalysis system," Applied Mathematical Modelling, vol. 39, no. 1, pp. 50-69, 2015.

[21] J. Xu, G. X. Yang, H. G. Xi, and J. Z. Su, "Pattern dynamics of a predator-prey reaction-diffusion model with spatiotemporal delay," Nonlinear Dynamics, vol. 81, Article ID 2155C2163, 2015.

[22] L. Guan and J. Shen, "Self-organized pattern dynamics of somitogenesis model in embryos," Physica A: Statistical Mechanics and Its Applications, vol. 506, pp. 587-601, 2018.

[23] Q. Ouyang, Introduction to Nonlinear Science and Pattern Dynamics, Peking University Press, Beijing, China, 2010.

[24] C. Mayol, R. Toral, and C. R. Mirasso, "Derivation of amplitude equations for nonlinear oscillators subject to arbitrary forcing," Physical Review E, vol. 69, no. 6, Article ID 066141, 2004.

[25] J. Murray, Mathematical Biology: I. An Introduction, Springer, Berlin, Germany, 2007.

[26] Y. Kuramoto, Chemical Oscillations, Waves and Turbulence, Dover Publications, Mineola, NY, USA, 2003. 
[27] D. Serre, Matrices: Theory and Applications, Springer, Berlin, Germany, 2002.

[28] G. H. Gunaratne, Q. Ouyang, and H. L. Swinney, "Pattern formation in the presence of symmetries," Physical Review E, vol. 50, no. 4, pp. 2802-2820, 1994.

[29] B. Pena and C. Perez-Garcia, "Stability of turing patterns in the brusselator model," Physical Review E, vol. 64, no. 5, Article ID 056213, 2001.

[30] V. A. Zorich, Mathematical Analysis, Springer, Berlin, Germany, 2004. 